\title{
Models of Sensor Operations for Border Surveillance
}

\author{
Roberto Szechtman Moshe Kress Kyle Lin Dolev Cfir
}

September 11, 2007

\begin{abstract}
This paper is motivated by the diverse array of border threats, ranging from terrorists to arms dealers and human traffickers. We consider a moving sensor that patrols a certain section of a border with the objective to detect infiltrators who attempt to penetrate that section. Infiltrators arrive according to a Poisson process along the border with a specified distribution of arrival location, and disappear a random amount of time after their arrival. The measures of effectiveness are the target (infiltrator) detection rate and the time elapsed from target arrival to target detection. We study two types of sensor trajectories that have constant endpoints, are periodic, and maintain constant speed: (1) a sensor that jumps instantaneously from the endpoint back to the starting-point, and (2) a sensor that moves continuously back and forth. The controlled parameters (decision variables) are the starting and end points of the patrolled sector and the velocity of the sensor. General properties of these trajectories are investigated.
\end{abstract}

\section{Introduction}

The problem of illegal cross-border activity, in particular along the U.S.-Mexico border, has drawn much attention in recent years [13], [7]. In addition to people who seek jobs and better life in the U.S., illegal border crossing has also been associated with criminal activities such as smuggling and drug trafficking. During the year 2000, over 1 million pounds of marijuana, 30,000 pounds of cocaine and 200 pounds of heroin were seized along the U.S.-Mexico border [6]. Thus, illegal border-crossing has been a cause for much concern to local, state and federal law enforcement authorities. Moreover, while so far there has not been any definite evidence of terrorists involvement associated with this illegal activity, the concern is that terrorists with weapons of mass destruction could possibly penetrate the porous border in much the same way that illegal migrants and smugglers do. This scenario poses a serious national security threat that must be handled by the authorities. While several stakeholders at all levels of government take part in securing the U.S. against such possible events, the most important stakeholder in border security is the U.S. Border Patrol, whose current primary mission is to prevent terrorists and terrorist weapons from entering the country [2]. This paper is motivated by that mission. 
Concerning border security, an increasingly common approach to border surveillance is to use a combination of long range and short range sensors [1]. Generally, there is a tradeoff between the range of the sensor and its resolution; long range sensors provide less resolution than short range sensors [1]. Although the models developed in this paper can be applied to a variety of surveillance systems, we focus on two systems: a mounted scanning camera that scans a certain sector of the border and then leaps back to the starting point, and a UAV-mounted sensor that flies back and forth between the two endpoints of the sector.

There are two different types of UAVs: drones and remotely piloted vehicles (RPVs). Both are pilot-less, but drones are programmed for autonomous flight, whereas RPVs are actively flown, remotely, by a ground control operator. UAVs have played key roles in recent conflicts [1], providing reconnaissance, surveillance, target acquisition, search and rescue, battle damage assessments and attack capabilities. An example of such a system that is increasingly present in U.S. border surveillance activities is the Predator B UAV system, which can provide long-endurance surveillance and communications relay [5]. While UAVs are used to extend the surveillance range, especially in the absence of ground infrastructure, ground-based electro-optical sensors achieve greater resolution. A variety of electro-optical imaging sensors are employed in border surveillance. A few examples of the technologies in use are high-resolution imaging, motion detection, temperature-differentiation and nightvision devices. In addition there is wide use of commercially available cameras adapted for daytime surveillance, infrared video (IR) detection systems and laser illumination systems that enable high-resolution imaging. Other optical components include computer-operated pan/tilt/zoom cameras, visible or near-infrared illuminators for night vision with conventional cameras and image-intensifiers for long-range night vision [5]. The combination of different sensors mitigates the shortcomings of each single sensor. For example, IR provides detection in atmospheric conditions where video imaging is ineffective, even though the resolution it provides is relatively low. Laser illumination sensors provide a combination of long range and high resolution images [5].

Previous work on linear patrols mainly concerns anti-submarine warfare. The classic WWII book by Koopman [9] lays the foundations for analyzing the performance of barrier patrols. Washburn, [18] and [19], extends the basic barrier patrol model. Models of barrier patrol have focused specifically on anti-submarine warfare, in which the acoustic degradation of the sonar sensor, caused by increasing the patroller's speed, is incorporated into the model, and the optimal patrol speed is found. A game-theoretic analysis of choice of speeds by infiltrator and patroller originated in [8] and [12], and was developed analytically for a continuum of speed choices in [10]. While these are important references, we are not aware of any past or current research that is applicable to our problem of perimeter protection. Although no queueing occurs in our models, some of the techniques we use resemble those employed in models based on spatial queues; see, for example, [3].

This paper sets forth models for a moving sensor that patrols a certain sector of a border. The goal is to find a patrol policy that minimizes the infiltration rate across that sector (maximize detections) when the sensor follows a periodic trajectory at constant speed. The controlled parameters (decision variables) of the trajectory are the beginning and end points of the patrolled sector and the sensor's velocity. We view the main contributions of this paper as follows. First, we develop models of border surveillance that capture the situation where infiltrators may disappear before sensor detection, and therefore traverse the border 
undetected. Second, we analyze and prove several properties of the optimal trajectories that are operationally significant. Finally, we provide a simulation analysis for the case where identifying the infiltrators is not instantaneous.

The type of surveillance systems described in this paper have recently been brought to public attention (see, for example, [17]) by the Department of Homeland Security, which signed a contract with the Boeing Corp. to secure the U.S. southern border. As part of this contract, tower-mounted and UAV-mounted sensors will be installed on the U.S.-Mexico border along parts of the state of Arizona.

The rest of the paper is organized as follows. Section 2 presents the basic framework of our models. Section 3 discusses properties of the leap-to-origin trajectory, and section 4 deals with the back-and-forth trajectory (see Figure 1). Section 5 extends the baseline scenario by considering the effect of sensor velocity and the case where it takes time to investigate each detected target.

\section{Model Preliminaries}

The two main components of the problem are a sensor and targets (infiltrators). We initially assume that the sensor is perfect and the detection and identification process is instantaneous. In particular, the detection probability is independent of the sensor velocity. We set the velocity, without loss of generality, to be 1 . These assumptions are relaxed later on. The sensor scans a certain sector of the border continuously and repetitively and our objective is to determine the endpoints of that sector-its origin and destination points. We assume that these points do not change during the surveillance mission. Two consecutive visits to the origin determine a cycle in the surveillance process. Targets arrive to the perimeter according to a homogeneous Poisson process with rate $\alpha$. More specifically, let $\left(T_{i}: i \geq 1\right)$ be the collection of arrival epochs, then the number of arrivals by the time $t, t \geq 0$ is given by

$$
N_{a}(t)=\sum_{i=1}^{\infty} I\left(T_{i} \leq t\right),
$$

where $I(\cdot)$ is the indicator function.

The arrival locations of the targets are described by a collection of independent and identically distributed (i.i.d.) continuous random variables $\left(X_{i}: i \geq 1\right)$ with common probability distribution $F_{X}$. After spending a certain time at the border area, a target may successfully infiltrate the border and therefore will no longer be available for detection. The period of time elapsed from target arrival until infiltration is the reneging time and is described by a collection $\left(R_{i}: i \geq 1\right)$ of i.i.d. non-negative continuous random variables with common distribution function $F_{R}$. Because targets may renege, the problem is to find the endpoints of the surveillance sector that represent the best tradeoff between two types of undetected (lost) targets: those who are outside the surveillance sector and those who renege within it.

The following assumption is satisfied in all practical situations of interest, and greatly simplifies the mathematical treatment.

Assumption 1 Throughout the paper we assume that random variables $\left(X_{i}: i \geq 1\right)$ posses a density $f_{X}$ that is unimodal and continuously differentiable on its support. We also assume 

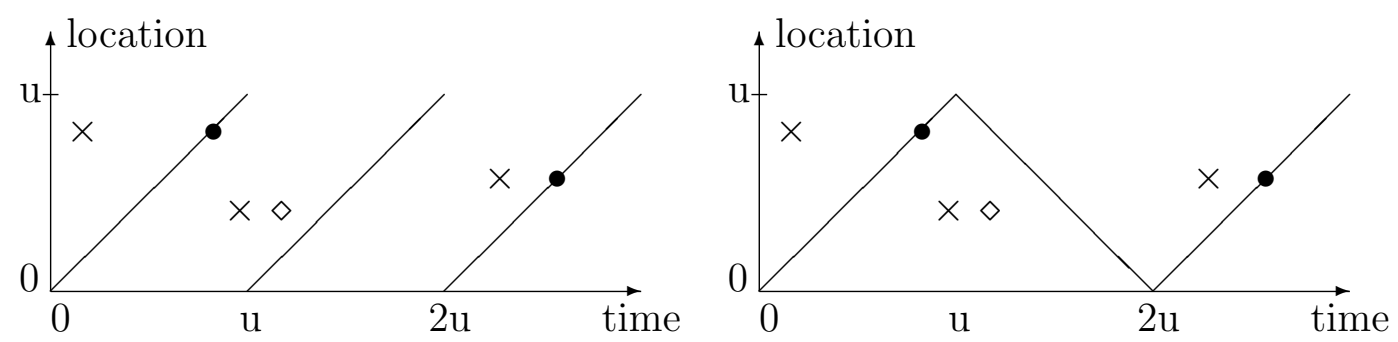

Figure 1: The LO trajectory (left) and the BF trajectory (right): Straight lines represent sensor's trajectories; the signs " $\times$ ", " $\bullet$ " and " $\diamond$ " represent arrival, detection and reneging of a target, respectively. Cycle length is $u$ in the left figure, and $2 u$ in the right figure.

that $F_{R}$ is continuously differentiable on its support.

We consider two types of generic sensors: A human-controlled remote video camera and a UAV-mounted sensor. A human-controlled remote camera scans the sector of the border [5] and is typically installed at the top of a tall tower. The camera starts the surveillance process at one end of the sector, moves at constant speed to a certain destination point, and then leaps back to the origin instantaneously. This is referred to as a leap-to-origin (LO) trajectory. A UAV-mounted sensor travels along the border sector back and forth in a constant speed, in a back-and-forth trajectory (BF). Figure 1 illustrates the LO scenario on the left-hand side and the BF scenario on the right-hand side.

The main component in our formulation is the process $\left(N_{d}(t): t \geq 0\right)$, which counts the number of detections by time $t$. Our construction guarantees that $N_{d}$ is a Poisson process for any cycle length $u$, with rate that depends on $F_{X}$ and $F_{R}$, on the sensor trajectory policy ( $\mathrm{LO}$ or $\mathrm{BF}$ ), and on the cycle length $u$. The measure of effectiveness (MOE) used to evaluate the $\mathrm{LO}$ and $\mathrm{BF}$ policies is the long term target detection rate, $N_{d}(t) / t$ as $t \rightarrow \infty$.

\section{The Leap-to-Origin Trajectory}

In this section we study the leap-to-origin (LO) trajectory, where the sensor instantaneously leaps from the destination to the origin after completing a cycle. Our goal is to find the two endpoints for the sensor to maximize the long-run detection rate. Recall that we assume that $N_{a}, X_{i}$, and $R_{i}$ are independent, that the $X_{i}$ 's have support on $\Re$, and that the $R_{i}$ 's have support on $\Re_{+}$. Without loss of generality (by changing the time scale if necessary) we assume that target arrival rate equals 1 . We also assume that the sensor speed equals 1 ; this assumption is relaxed later on in Section 5 .

The problem of finding the best endpoints is approached as follows:

(i) Identify the best origin point as a function of cycle length.

(ii) Find the optimal cycle length.

With regard to (i), define

$$
H(u):=\max _{a}\left\{F_{X}(u+a)-F_{X}(a)\right\}
$$


and let $a^{*}$ be a maximizer in (1). Observe that for $u$ arbitrary and finite, $F_{X}(u+a)-F_{X}(a) \rightarrow$ 0 as $|a| \rightarrow \infty$, so that $-\infty<a^{*}<\infty$. For a cycle length $u, a^{*}$ is the optimal origin of the sensor, $a^{*}+u$ is the optimal destination, and $H(u)$ is the probability that a target crosses the perimeter on the sector $\left(a^{*}, a^{*}+u\right)$; notice that $H(u)$ is a distribution function when viewed as a function of $u$. Moreover, the first part of Assumption 1 guarantees that $H(u)$ is sufficiently smooth for our models to be well posed.

Lemma 1 Under Assumption 1, H(u) is continuously differentiable and concave on its support.

Lemma 1 follows almost immediately from the next result.

Lemma 2 Suppose Assumption 1 holds. Then if $f_{X}$ has infinite support, the function a: $\Re \rightarrow \Re$ defined as the solution of

$$
f_{X}(a(u))=f_{X}(a(u)+u)
$$

is differentiable and $a(u)$ maximizes $F_{X}(u+a)-F_{X}(a)$.

Proof of Lemma 2. The implicit function theorem shows that $a(u)$ is well defined and differentiable. The first order necessary conditions for unconstrained optimization show that $F_{X}^{\prime}(a+u)-F_{X}^{\prime}(a)=0 \Longleftrightarrow f_{X}(a)=f_{X}(a+u)$ and, since $f_{X}$ is unimodal, there is only value of $a$ that makes $f_{X}(a)$ equal to $f_{X}(a+u)$ for $u$ given. $\otimes$

Proof of Lemma 1. If $f_{X}$ has infinite support, then

$$
H^{\prime}(u)=f_{X}(a(u)+u)\left(a^{\prime}(u)+1\right)-f_{X}(a(u)) a^{\prime}(u)=f_{X}(a(u)+u),
$$

by Lemma 2. Therefore, $H^{\prime}$ is continuous and non-increasing, and the lemma is proven for the infinite support situation. Suppose now that $f_{X}$ has finite support from the left; that is $\ell=\sup \left\{u: F_{X}(u)=0\right\}$ is finite. Define $u_{\ell}$ as the cycle length where the optimal trajectory origin first hits $\ell$. Therefore, for $0<u \leq u_{\ell}$ we have already shown that $H(u)$ is continuously differentiable and concave; whereas for $u>u_{\ell}$ we have $H(u)=F_{X}(\ell+u)-F_{X}(\ell)$, which is easily seen to be concave and continuously differentiable. The cases where $f_{X}$ has finite support from the right, and from the left and the right, are similarly treated. $\otimes$

A target is detected on the $k+1$ 'th cycle, for $k \geq 1$, when all of the following three conditions are met: First, the target arrives to the patrolled sector $\left(a^{*} \leq X_{i} \leq a^{*}+u\right)$; second, its arrival time is at least $X_{i}-a^{*}+(k-1) u$ (since we assume that sensor velocity is 1) and no more than $X_{i}-a^{*}+k u$; last, its reneging time is at least $X_{i}-a^{*}+k u-T_{i}$. This situation is illustrated in Figure 2, for $k=2$ : The first two conditions determine the dotted region where detection is possible; the last condition ensures that a target that falls in the dotted area has a reneging time sufficiently large to be detected.

Let $X_{i}^{*}:=X_{i}-a^{*}$ be the target arrival location translated to $a^{*}$, for $i=1,2, \ldots$ Hence, for an LO trajectory with origin $a^{*}$ and destination $a^{*}+u$, the expression

$$
N_{d}((k+1) u)=N_{d}(k u)+\sum_{i=1}^{\infty} I\left(0 \leq X_{i}^{*} \leq u ; X_{i}^{*}+(k-1) u \leq T_{i} \leq X_{i}^{*}+k u ; R_{i}>X_{i}^{*}+k u-T_{i}\right)
$$




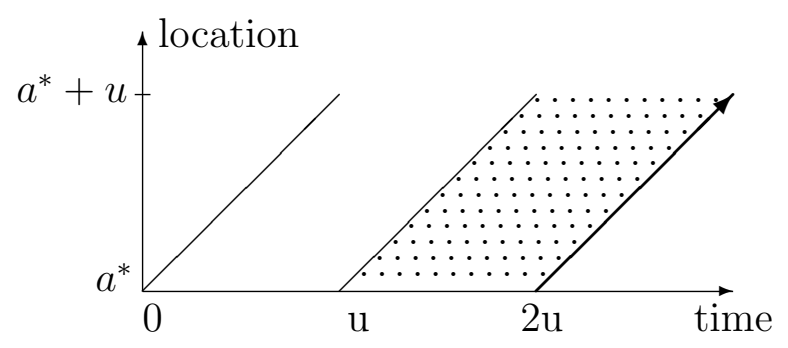

Figure 2: Detection region for the third cycle of an LO trajectory, for cycle length $u$, and optimal origin of the sensor $a^{*}$.

represents the number of detections at the end of the $k+1$ 'th cycle, for $k \geq 1$. Observe that since the first cycle is irregular, we have that

$$
N_{d}(u)=\sum_{i=1}^{\infty} I\left(0 \leq X_{i}^{*} \leq u ; T_{i} \leq X_{i}^{*} ; R_{i}>X_{i}^{*}-T_{i}\right)
$$

is the number of detections at the end of the first cycle.

We wish to choose a cycle length and an origin that maximize the detection rate $N_{d}(t) / t$, as $t \rightarrow \infty$. The following result, Proposition 4.4.1 in [14], provides theoretical support for the computation of this rate.

Proposition 1 Suppose that $\left(Y_{i}: i \geq 1\right)$ are random elements of an Euclidean space $E_{1}$ such that $N(\cdot)=\sum_{i} I\left(Y_{i} \in \cdot\right)$ is a Poisson process with Poisson measure $\mu(\cdot)=E N(\cdot)$. Suppose $\left(J_{i}: i \geq 1\right)$ are random elements of a second Euclidean space $E_{2}$ with common probability distribution $F$, and suppose $N$ and the $J_{i}$ 's are defined on the same probability space and are independent. Then the point process $\sum_{i} I\left(\left(Y_{i}, J_{i}\right) \in \cdot\right)$ is Poisson with Poisson measure $\mu \times F$.

Proposition 1 guarantees that the process $N_{d}$ is Poisson. The property of the Poisson process that disjoint intervals are independent implies that the random variables that describe the number of detections in a regular cycle are independent and identically distributed, with mean given by

$$
\begin{aligned}
E & N_{d}((k+1) u)-E N_{d}(k u) \\
& =\int_{\Re} \int_{\Re_{+}} \int_{\Re_{+}} I\left(0 \leq x^{*} \leq u ; x^{*}+(k-1) u \leq t \leq x^{*}+k u ; r>x^{*}+k u-t\right) d F_{R}(r) d t d F_{X^{*}}\left(x^{*}\right) \\
& =\int_{0}^{u} \int_{x^{*}+(k-1) u}^{x^{*}+k u} \bar{F}_{R}\left(x^{*}+k u-t\right) d t d F_{X^{*}}\left(x^{*}\right) \\
& =H(u) \int_{0}^{u} \bar{F}_{R}(t) d t
\end{aligned}
$$

for $k \geq 1$, where $\bar{F}_{R}(\cdot)=1-F_{R}(\cdot)$, and $F_{X^{*}}\left(x^{*}\right)=P\left(X^{*} \leq x^{*}\right)$. Observe that although $N_{d}$ does not necessarily have a constant rate within a cycle, the rate is periodic for all cycles 
$k \geq 2$, and hence we end up with the above result. For the first irregular cycle we have

$$
E N_{d}(u)=\int_{\Re} \int_{\Re_{+}} \int_{\Re_{+}} I\left(0 \leq x^{*} \leq u ; 0 \leq t \leq x^{*} ; r>x^{*}-t\right) d F_{R}(r) d t d F_{X^{*}}\left(x^{*}\right)<\infty .
$$

Equation (2) and the monotone convergence Theorem imply that $N_{d}(u) / t \rightarrow 0$ almost surely as $t \rightarrow \infty$. Also, the Law of Large Numbers for Renewal Reward processes, Proposition 3.4.1 in [14], implies that

$$
\frac{N_{d}(t)-N_{d}(u)}{t-u} \frac{t-u}{t} \rightarrow \frac{E N_{d}((k+1) u)-E N_{d}(k u)}{u}
$$

almost surely as $t \rightarrow \infty$. Putting this all together,

$$
\begin{aligned}
\frac{N_{d}(t)}{t} & =\frac{N_{d}(u)}{t}+\frac{N_{d}(t)-N_{d}(u)}{t-u} \frac{t-u}{t} \\
& \rightarrow \frac{E N_{d}((k+1) u)-E N_{d}(k u)}{u},
\end{aligned}
$$

almost surely as $t \rightarrow \infty$, for any $k \geq 1$. Therefore, the detection rate is

$$
g_{L O}(u):=H(u) \frac{\int_{0}^{u} \bar{F}_{R}(t) d t}{u} .
$$

Accordingly, our goal in the LO setting is to find a cycle length that maximizes $g_{L O}$. Just like in (1) when selecting the best origin $a(u)$, there may exist more than one maximizer of $g_{L O}$ in $(3)$, in which case we choose the one with the smallest cycle length. That is, optimal cycle length is

$$
u^{*}:=\min \left\{u \geq 0: g_{L O}(u) \geq g_{L O}(w), \forall w \geq 0\right\} .
$$

The term $\int_{0}^{u} \bar{F}_{R}(t) d t$ in (3) is the expected number of detections in any inspected location in a cycle of length $u$, which is then inflated by $H(u)$ to account for all surveyed locations; the $u$ term in the denominator is the expected number of arrivals along a cycle of length $u$.

Remark 1 Changing the arrival rate of $N_{a}$ from 1 to $\alpha>0$ changes $g_{L O}$ by a factor $\alpha$, but does not change $a^{*}$ nor $u^{*}$.

We define $g_{L O}(0)=0$, so that $g_{L O}$ is continuous at 0 . Observe that Assumption 1 implies $g_{L O}$ is continuously differentiable in $\Re_{+}$. To complete the notation, let $\bar{r}:=\inf \left\{\nu: F_{R}(\nu)=\right.$ $1\}$, and $\bar{u}:=\inf \{\nu: H(\nu)=1\}$ (both $\bar{r}$ and $\bar{u}$ can possibly be infinite); and write $\mu_{R}=E R$ and $\mu_{X}=E X$. Given a differentiable real function $\ell(u)$ we write $\ell^{\prime}(u)$ as short for $d \ell(u) / d u$.

We study properties of the detection rate and the optimal cycle length in various scenarios of the LO trajectory. First we study several instances of reneging behavior: $R$ bounded with probability one, $R$ deterministic, and $R$ uniformly distributed. Second, we analyze the case of $X$ bounded, and $X$ uniform. Last, we deal with the general case where both $X$ and $R$ have densities with infinite support. We start by analyzing the case where the reneging times are bounded with probability 1. 
Example 1 Suppose $0<\bar{r}<\infty$. Then

$$
g_{L O}(u)=\left\{\begin{array}{c}
H(u) \frac{\int_{0}^{u} \bar{F}_{R}(t) d t}{u}, \text { for } 0 \leq u \leq \bar{r} \\
H(u) \frac{\mu_{R}}{u}, \text { for } u>\bar{r} .
\end{array}\right.
$$

Evidently, if $g_{L O}$ is non-increasing on $[\bar{r}, \infty)$, we must have $u^{*} \leq \bar{r}$.

Lemma 3 Suppose that $0<\bar{r}<\infty$. Then, $u^{*} \leq \bar{r}$.

Proof: Lemma 1 implies $u H^{\prime}(u) \leq H(u)$ for $u \geq \bar{r}$, so that $(H(u) / u)^{\prime} \leq 0$, and $g_{L O}^{\prime}(u) \leq 0$. $\otimes$

Clearly, $H$ strictly concave implies $u H^{\prime}(u)<H(u)$. The borderline case is $u H^{\prime}(u)=H(u)$ for $u \geq \bar{r}$, that is, $H^{\prime}$ constant on $u \geq \bar{r}$; see Example 4 below. What happens if $u H^{\prime}(u)>H(u)$ for some $u \geq \bar{r}$ ? Then it could happen that $u^{*}>\bar{r}$. In any case, because $H(u) / u \rightarrow 0$ as $u \rightarrow \infty$, we know that $u^{*}<\infty$.

Under the conditions of Lemma 3 it is never beneficial to scan a sector longer than $\bar{r}$ because the detections gained by covering more area would not outweight the extra missed detections due to reneging. Two special cases of Example 1 are presented next.

Example 2 Deterministic reneging times: Suppose $P(R=\bar{r})=1,0<\bar{r}<\infty$. Then Equation (4) becomes

$$
g_{L O}(u)=\left\{\begin{array}{c}
H(u), \text { for } 0 \leq u \leq \bar{r} \\
H(u) \frac{\bar{r}}{u}, \text { for } u>\bar{r} .
\end{array}\right.
$$

Since $g_{L O}$ is non-decreasing on $0 \leq u \leq \bar{r}$, in view of Lemma 3 we have $u^{*}=\bar{r}$.

Example 3 If $R_{i} \sim U(0, \bar{r})$, Equation (4) becomes

$$
g_{L O}(u)=\left\{\begin{array}{c}
H(u)\left(1-\frac{u}{2 \bar{r}}\right), \text { for } 0 \leq u \leq \bar{r} \\
H(u) \frac{\bar{r}}{2 u}, \text { for } u>\bar{r} .
\end{array}\right.
$$

Therefore, $u^{*} \leq \bar{r}$, where $u^{*}$ is the smallest maximizer of the top branch in the RHS of (5).

Our next lemma considers the case of $\bar{u}$ finite.

Lemma 4 Suppose that $0<\bar{u}<\infty$. Then $u^{*} \leq \bar{u}$.

Proof: The fact that $\int_{0}^{u} \bar{F}_{R}(t) d t / u$ is non-increasing in $u$, and that $H(u)=1$ for all $u \geq \bar{u}$, implies that $u^{*} \leq \bar{u}$. $\otimes$

Lemma 4 describes the scenario corresponding to $X_{i}$ having its mass concentrated in some finite interval. It says that it is not beneficial to patrol further than $\bar{u}$, where no targets arrive. Our next example illustrates Lemma 4, and shows that the hypothesis of Lemma 3 cannot be weakened.

Example 4 Suppose $X_{i} \sim U(0, \bar{u})$. Then Equation (3) becomes

$$
g_{L O}(u)=\left\{\begin{array}{c}
\frac{\int_{0}^{u} \bar{F}_{R}(t) d t}{\bar{u}_{\bar{u}}}, \text { for } 0 \leq u \leq \bar{u} \\
\frac{\int_{0}^{\bar{u}} \bar{F}_{R}(t) d t}{u}, \text { for } u>\bar{u} .
\end{array}\right.
$$

Clearly, $u^{*}=\bar{u}$. In particular, if $\bar{r}<\bar{u}$, then any cycle length in $[\bar{r}, \bar{u}]$ maximizes $g_{L O}$. 
We now discuss properties of $g_{L O}(u)$ and $u^{*}$ when both $X$ and $R$ have densities with infinite support. First, it is immediate from the definition of $g_{L O}$ that $g_{L O}(u) u \rightarrow \mu_{R}$ as $u \rightarrow \infty$, so that $g_{L O}(u)$ decreases towards 0 at rate $1 / u$; see the right part of Figure 5 . Concerning the behavior of $g_{L O}$ locally around 0 we observe that $g_{L O}$ is sub-additive on some interval $(0, \gamma], \gamma>0$ :

$$
g_{L O}(\alpha+\beta) \leq g_{L O}(\alpha)+g_{L O}(\beta),
$$

for $\alpha, \beta>0$ and $\alpha+\beta \leq \gamma$. Sub-additivity follows because

(i) $H(\alpha+\beta) \leq H(\alpha)+H(\beta)$. This follows since

$$
\begin{aligned}
H(\alpha+\beta) & =\max _{a}\left\{F_{X}(\alpha+\beta+a)-F_{X}(a)\right\} \\
& =\max _{a}\left\{F_{X}(\alpha+\beta+a)-F_{X}(\alpha+a)+F_{X}(\alpha+a)-F_{X}(a)\right\} \\
& \leq \max _{a}\left\{F_{X}(\alpha+\beta+a)-F_{X}(\alpha+a)\right\}+\max _{a}\left\{F_{X}(\alpha+a)-F_{X}(a)\right\} \\
& =\max _{a}\left\{F_{X}(\beta+a)-F_{X}(a)\right\}+\max _{a}\left\{F_{X}(\alpha+a)-F_{X}(a)\right\} \\
& =H(\beta)+H(\alpha) .
\end{aligned}
$$

(ii) $\int_{0}^{\alpha+\beta} \bar{F}_{R}(t) d t /(\alpha+\beta) \leq \min \left\{\int_{0}^{\alpha} \bar{F}_{R}(t) d t / \alpha, \int_{0}^{\beta} \bar{F}_{R}(t) d t / \beta\right\}$. This follows because $\int_{0}^{u} \bar{F}_{R}(t) d t / u$ is non-increasing in $u$, as can be immediately seen by evaluating its first derivative.

Applying properties (i) and (ii) leads immediately to (6):

$$
\begin{aligned}
g_{L O}(\alpha+\beta) & =\frac{H(\alpha+\beta) \int_{0}^{\alpha+\beta} \bar{F}_{R}(t) d t}{\alpha+\beta} \\
& \leq(H(\alpha)+H(\beta)) \min \left\{\frac{\int_{0}^{\alpha} \bar{F}_{R}(t) d t}{\alpha}, \frac{\int_{0}^{\beta} \bar{F}_{R}(t) d t}{\beta}\right\} \\
& \leq H(\alpha) \frac{\int_{0}^{\alpha} \bar{F}_{R}(t) d t}{\alpha}+H(\beta) \frac{\int_{0}^{\beta} \bar{F}_{R}(t) d t}{\beta} \\
& =g_{L O}(\alpha)+g_{L O}(\beta) .
\end{aligned}
$$

In addition, $g_{L O}$ is continuous at 0 . Therefore, Lemma 3.6.4 of [4] applies, and we have

$$
\frac{g_{L O}(u)}{u} \rightarrow \lambda
$$

as $u \rightarrow 0$, for some $\lambda>0$. That is, $g_{L O}$ is locally linear around 0 ; see the left part of Figure 5. Thus, the behavior of $g_{L O}(u)$ for $u$ around 0 and for all $u$ sufficiently large implies that the optimal cycle length must be a stationary point, i.e.: $u^{*}$ is the root of the equation $g_{L O}^{\prime}(u)=0$ for which $g_{L O}$ is largest. The root problem can be re-written as

$$
u H^{\prime}(u) \int_{0}^{u} \bar{F}_{R}(t) d t=H(u) \int_{0}^{u} \bar{F}_{R}(t) d t-u H(u) \bar{F}_{R}(u) .
$$


The last equation says that we increase cycle length until the marginal increase in target detections equals the marginal decrease in lost target detections due to reneging. In general, however, it is not possible to analytically solve Equation (7), so standard numerical methods must be utilized; the computational cost grows with the number of roots that need to be found in (7).

Example 5 Suppose $X_{i} \sim N(0,1)$ and $R_{i} \sim \exp (1)$. Then $a^{*}=-1.025, u^{*}=2.05$ is the root of $g_{L O}^{\prime}(u)=0$, and $g_{L O}\left(u^{*}\right)=.295$; see Figure 5 .

Remark 2 Concerning Example 5, an exponential reneging distribution leads to the same solution that arises in the following setting: Upon arrival, each target emits a signal of constant amplitude that decays exponentially in time with rate $\theta$; this is known as a shot noise process [14]. This would be the case, for example, if the footprint and marks left by the infiltrators disappear at an exponential rate in time. In particular, the signal strength of arrivals detected in the $(k+1)$ 'th cycle is given by

$$
\sum_{i=1}^{\infty} e^{-\theta\left(X_{i}+k u-T_{i}\right)} I\left(a^{*} \leq X_{i} \leq a^{*}+u, X_{i}+(k-1) u \leq T_{i} \leq X_{i}+k u\right) .
$$

Following the approach taken in [14], p. 326, we find the expected average signal strength of targets detected in an arbitrary cycle is $H(u) \int_{0}^{u} \exp (-\theta t) d t / u$, which is $g_{L O}(u)$ when $R_{i} \sim \exp (\theta)$. In other words, a system where targets that arrive according to a shot noise process, has the same solution as the system where targets renege according to an exponential distribution.

Next, we study the effect of variability in the targets' arrival location. The question we have in mind is: Does an increase in $\sigma_{X}^{2}:=\operatorname{var}(X)$ cause $g_{L O}$ to decrease? The answer is not necessarily, as can be seen in the following example.

Example 6 Suppose that $X$ has a density that is a mixture of a uniform and a Pareto density,

$$
f_{X}(x)=\left\{\begin{array}{c}
1, \text { for } 0 \leq x \leq \theta \\
(1-\theta) \frac{\theta}{x^{2}}, \text { for } x>\theta
\end{array}\right.
$$

and that $P(R \leq \theta)=1$, for $0<\theta<1$. It is easy to see that $H(u)=1+\left(\theta^{2}-\theta\right) / u$ for $u \geq \theta$, and that $\theta>1 / 2$ implies $(H(u) / u)^{\prime}<0$ for $u>\theta$. Therefore, according to Lemma 3 and Example 4 we have $u^{*}=\theta$, and $g_{L O}\left(u^{*}\right)=\mu_{R}$. Compare this with the case $X_{i} \sim U(0, \bar{u})$, where $\bar{u}>\theta$. In this case we have, according to Example $4, u^{*}=\theta$ and $g_{L O}\left(u^{*}\right)=\mu_{R} / \bar{u}$. Therefore, if $\bar{u}>1$, the optimal detection rate for the mixture density case is larger than that of the non-uniform case. However, $\sigma_{X}^{2}$ in the mixture case is infinite (due the heavy-tails of the Pareto density), while $\sigma_{X}^{2}$ in the second case is finite. This example lets us conclude that larger arrival location variability does not necessarily lead to lower detection rate for the optimal cycle length.

However, for re-scaled versions of $X$ it is true that lower variance leads to greater detection rate. To show this it suffices to compare the respective $H(\cdot)$ terms. 
Lemma 5 Let $\tilde{X}=X / \sigma_{X}$, $\tilde{a}^{*}$ a maximizer of $P(a<\tilde{X} \leq a+u)$, and $\tilde{H}(u)=P\left(\tilde{a}^{*}<\tilde{X} \leq\right.$ $\left.\tilde{a}^{*}+u\right)$. Suppose $0<\sigma_{X}<\infty$. Then $\tilde{H}(u) \leq H(u)$ if and only if $\sigma_{X} \leq 1$.

Proof: $\quad$ For $\sigma_{X}>1$ we have $\tilde{H}(u)=P\left(\sigma_{X} \tilde{a}^{*}<X \leq \sigma_{X} \tilde{a}^{*}+\sigma_{X} u\right) \geq P\left(a^{*}<X \leq\right.$ $\left.a^{*}+\sigma_{X} u\right) \geq P\left(a^{*}<X \leq a^{*}+u\right)=H(u)$. On the other hand, for $\sigma_{X}<1$ we have $\tilde{H}(u) \leq P\left(\sigma_{X} \tilde{a}^{*}<X \leq \sigma_{X} \tilde{a}^{*}+u\right) \leq P\left(a^{*}<X \leq a^{*}+u\right)=H(u) . \otimes$

Example 7 Suppose $X_{i} \sim N\left(0, \sigma^{2}\right)$, and $R_{i} \sim \exp (1)$. The top of Figure 3 illustrates the optimal detection rate as a function of $\sigma$; as expected from Lemma $5, g_{L O}\left(u^{*}\right)$ decreases as $\sigma$ increases. The bottom of Figure 3 shows the optimal cycle length increasing with $\sigma$. Not shown is the optimal origin $a^{*}=-u^{*} / 2$.
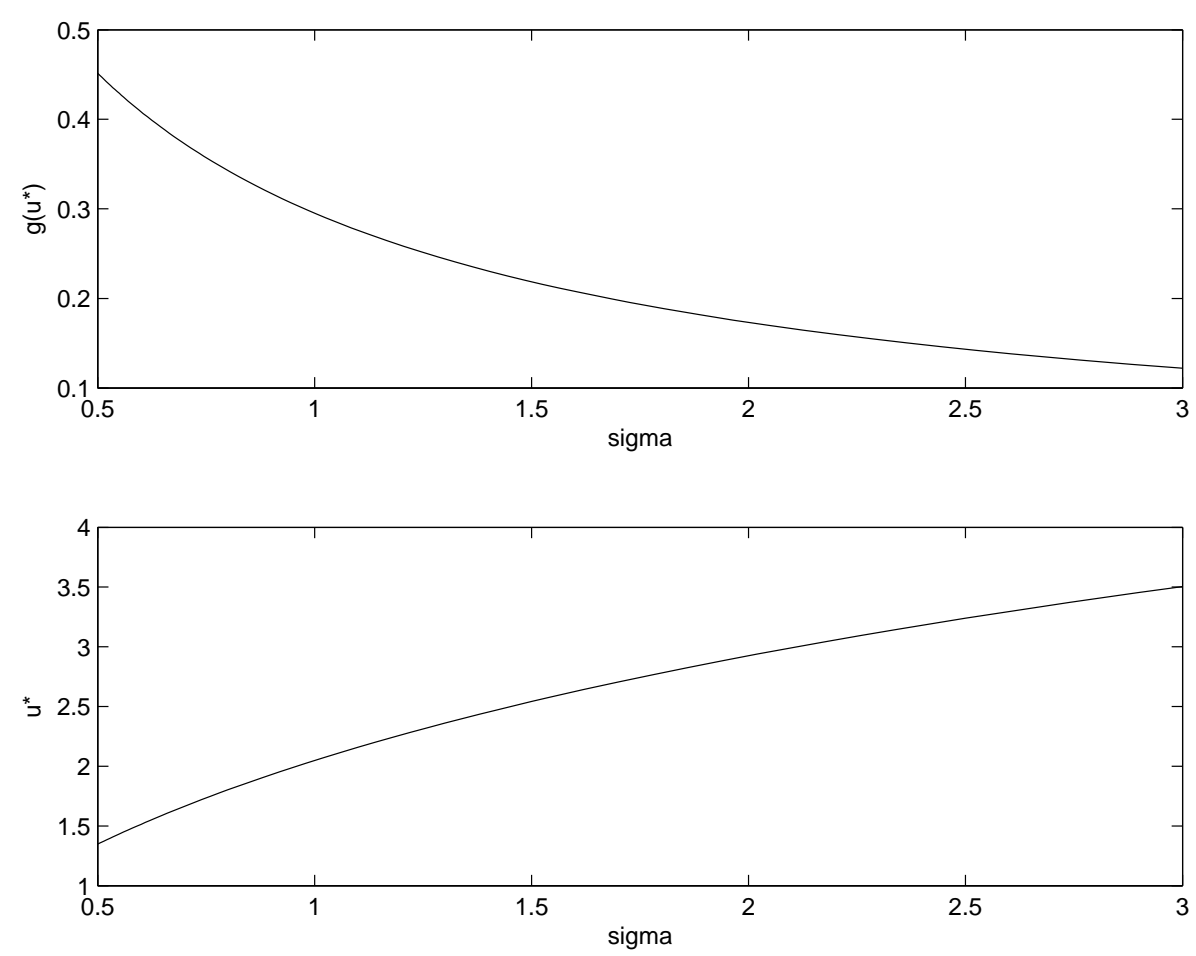

Figure 3: Detection rate and cycle length as a function of $\sigma$.

\section{The Back-and-Forth Trajectory}

In this section we study the back-and-forth $(\mathrm{BF})$ trajectory - a scenario that arises when sensors are mounted on platforms, such as ground patrol units or UAVs. Our goal is to analyze cycle trajectories and find an optimal cycle length in order to maximize the long-run detection rate for targets before they renege.

The setting is the same as that in the LO scenario: $X_{i}, R_{i}$, and $N_{a}(\cdot)$ are mutually independent; the $X_{i}$ 's and $R_{i}$ 's are continuous random variables whose distributions satisfy Assumption 1; and the $R_{i}$ 's take values on $\Re_{+}$. The speed of the sensor is 1 , and the targets 


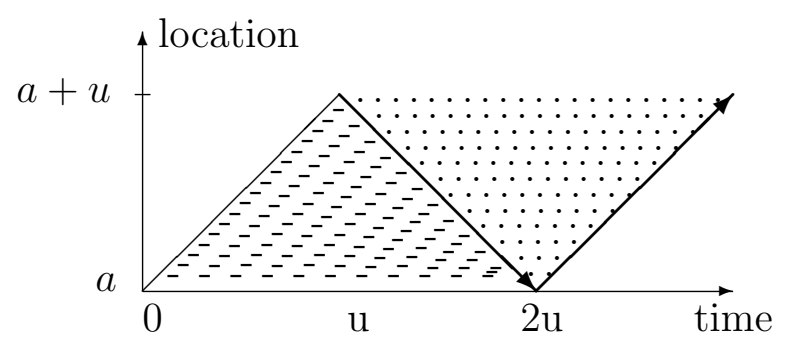

Figure 4: Detection region for the first regular cycle of a BF trajectory, for cycle length $2 u$, and origin of the sensor $a$.

arrive according to a Poisson process with rate 1. As shown in Figure 1, because the re-visit time to the center of the trajectory is shorter than that of the endpoints, targets renege more often in an area closer to the trajectory endpoints.

In order to obtain an expression for the long-run detection rate, we take a similar approach to the one taken to determine $g_{L O}$. Consider Figure 4: We filled with "-" marks the region where targets get detected (if no reneging occurs) when the sensor moves from $a+u$ to $u$, and we filled with "." marks the region where targets get detected (if no reneging occurs) when the sensor moves from $u$ back to $a+u$. The cycle length is $2 u$, and $a$ is the cycle origin. For a target $i$ to fall in the "-" marked region and get detected it must satisfy three conditions: $a \leq X_{i} \leq a+u, X_{i}-a \leq T_{i} \leq 2 u-\left(X_{i}-a\right)$, and $R_{i}>2 u-T_{i}-\left(X_{i}-a\right)$. On the other hand, for a target to fall in the "." marked region and get detected, it must satisfy: $a \leq X_{i} \leq a+u, 2 u-\left(X_{i}-a\right) \leq T_{i} \leq 2 u+\left(X_{i}-a\right)$, and $R_{i}>2 u-T_{i}+\left(X_{i}-a\right)$. A similar analysis can be carried out for all the regular cycles, by introducing a cycle-index $k$, just like we did in the LO case. Hence, Proposition 1 ensures that the detection process for the BF trajectory is Poisson, so that the number of detections in each regular cycle is a sequence of independent and identically distributed random variables, with the mean for each cycle given by

$$
\begin{aligned}
\int_{a}^{a+u} \int_{x-a}^{2 u-(x-a)} & \bar{F}_{R}(2 u-(x-a)-t) d t d F_{X}(x)+\int_{a}^{a+u} \int_{2 u-(x-a)}^{2 u+(x-a)} \bar{F}_{R}(2 u-t+(x-a)) d t d F_{X}(x) \\
& =\int_{a}^{a+u} \int_{0}^{2(u-(x-a))} \bar{F}_{R}(t) d t d F_{X}(x)+\int_{a}^{a+u} \int_{0}^{2(x-a)} \bar{F}_{R}(t) d t d F_{X}(x) \\
& =\int_{0}^{u} f_{X}(x+a) c(x, u) d x
\end{aligned}
$$

where $f_{X}$ is the density of the $X_{i}$ 's and

$$
c(x, u):=\int_{0}^{2(u-x)} \bar{F}_{R}(t) d t+\int_{0}^{2 x} \bar{F}_{R}(t) d t,
$$

for $0 \leq x \leq u$.

Since the mean number of detections in the first irregular cycle is finite, the Law of Large Numbers for Renewal Reward processes ensures that the long-run detection rate for targets 
is given by

$$
g_{B F}(u):=\frac{\max _{a}\left\{\int_{0}^{u} f_{X}(x+a) c(x, u) d x\right\}}{2 u} .
$$

The constant $a_{B F}^{*}=a(u)$ maximizes $\int_{0}^{u} f_{X}(x+a) c(x, u) d x$, and is the best trajectory origin for cycle length $u$. Since $f(x+a) \rightarrow 0$ as $|a| \rightarrow \infty$ and $c(x, u) \leq 2 \mu_{R}$ for $0 \leq x \leq u$, we must have $\left|a^{*}\right|<\infty$. From Equation (8), it can be seen that the function $c(\cdot, u)$ is symmetric for each $u>0$ about $x=u / 2$. In addition, differentiation with respect to $x$ shows that $c(\cdot, u)$ is increasing and concave in $x$ for fixed $u$. Define $g_{B F}(0)=0$ so that $g_{B F}$ is continuous in $\Re_{+}$, and observe that Assumption 1 implies that $g_{B F}$ is continuously differentiable in $\Re_{+}$(the proof of this fact mimics that of Lemma 1). The optimal cycle length is the smallest cycle length that maximizes $g_{B F}$ :

$$
u_{B F}^{*}:=\min \left\{u \geq 0: g_{B F}(u) \geq g_{B F}(w), \forall w \geq 0\right\},
$$

The analysis in this section is similar to that of the LO trajectory, and we draw comparisons between $\mathrm{LO}$ and $\mathrm{BF}$ whenever appropriate. In this regard, the next lemma shows that the BF trajectory can do no better than the LO trajectory, for any cycle length $u$.

Lemma 6 For all $u \geq 0, g_{B F}(u) \leq g_{L O}(u)$.

Proof: Observe that $\bar{F}_{R}$ non-increasing implies that $c(x, u) \leq 2 \int_{0}^{u} \bar{F}_{R}(t) d t$, for all $0 \leq x \leq$ $u$. Hence

$$
g_{B F}(u)=\frac{\max _{a}\left\{\int_{0}^{u} f_{X}(x+a) c(x, u) d x\right\}}{2 u} \leq \frac{\max _{a}\left\{\int_{0}^{u} f_{X}(x+a) d x\right\} \int_{0}^{u} \bar{F}_{R}(t) d t}{u}=g_{L O}(u) .
$$

$\otimes$

In addition,

\section{Corollary 1 Lemma 6 implies}

$$
g_{B F}\left(u_{B F}^{*}\right) \leq g_{L O}\left(u_{B F}^{*}\right) \leq g_{L O}\left(u_{L O}^{*}\right),
$$

that is, the optimal detection rate for the LO trajectory cannot be smaller than the optimal detection rate for the BF trajectory.

We now focus on properties of the optimal cycle length $u_{B F}^{*}$. We start with the analogue of Lemma 3, dealing with the case of $R_{i}$ having finite support, $P\left(R_{i} \leq \bar{r}\right)=1$, for $0<\bar{r}<\infty$. The key idea is that for $u \gg \bar{r}, g_{B F}(u) \approx g_{L O}(u)$. Specifically, for $u \geq \bar{r}$ we have

$$
g_{B F}(u)=\mu_{R} \frac{F_{X}\left(u+a_{B F}^{*}\right)-F_{X}\left(a_{B F}^{*}\right)-\varphi(u)}{u},
$$

(cf. Equation (4)) where

$$
\begin{aligned}
& \varphi(u):= \\
& \frac{\int_{0}^{\bar{r} / 2} f_{X}\left(x+a_{B F}^{*}\right)\left(\mu_{R}-\int_{0}^{2 x} \bar{F}_{R}(t) d t\right) d x+\int_{u-\bar{r} / 2}^{u} f_{X}\left(x+a_{B F}^{*}\right)\left(\mu_{R}-\int_{0}^{2(u-x)} \bar{F}_{R}(t) d t\right) d x}{2 \mu_{R}}
\end{aligned}
$$

is the "error" term. 
Lemma 7 Suppose that $0<\bar{r}<\infty$, that $g_{B F}$ is differentiable in $\Re_{+}$, and that the error term $\varphi(u)$ satisfies

$$
u\left(f_{X}\left(u+a_{B F}^{*}\right)-\varphi^{\prime}(u)\right) \leq F_{X}\left(u+a_{B F}^{*}\right)-F_{X}\left(a_{B F}^{*}\right)-\varphi(u),
$$

for all $u \geq \bar{r}$. Then $u_{B F}^{*} \leq \bar{r}$.

Proof: The hypothesis implies that $g_{B F}^{\prime}(u) \leq 0$ for all $u \geq \bar{r}$. $\otimes$

Remark 3 Condition (11) is the same as that of Lemma 3, but for the error term $\varphi$. The error term becomes insignificant as $\bar{r}$ becomes large, but may be relevant for small values of $\bar{r}$-see Example (8) below.

Example 8 When $P(R=\bar{r})=1$ for some constant $\bar{r}>0$ we have

$$
c(x, u)=\min \{2 x, \bar{r}\}+\min \{2(u-x), \bar{r}\} .
$$

For $u \leq \bar{r} / 2$ we have $c(x, u)=2 u$, and $g_{B F}(u)=F_{X}\left(u+a_{B F}^{*}\right)-F_{X}\left(a_{B F}^{*}\right)$ non-decreasing implies $\bar{r} / 2 \leq u_{B F}^{*}$. If the conditions of Lemma 7 apply, we have $\bar{r} / 2 \leq u_{B F}^{*} \leq \bar{r}$.

The analogue of Lemma 4 to the BF setting is straightforward.

Lemma 8 Suppose $P(\underline{u} \leq X \leq \bar{u})=1$, where $\underline{u}=\sup \{u: P(X \geq u)=1\}$ and $\bar{u}=\inf \{u$ : $P(X \leq u)=1\}$, and $-\infty<\underline{u}<\bar{u}<\infty$. Then $u_{B F}^{*} \leq \bar{u}-\underline{u}$.

Proof: For $u_{+} \geq \bar{u}$ and $u_{-} \leq \underline{u}$, we have

$$
\frac{\int_{u_{-}}^{u_{+}} f_{X}(x) c(x, u) d x}{2\left(u_{+}-u_{-}\right)}=\frac{\int_{\underline{u}}^{\bar{u}} f_{X}(x) c(x, u) d x}{2\left(u_{+}-u_{-}\right)} \leq \frac{\int_{\underline{u}}^{\bar{u}} f_{X}(x) c(x, u) d x}{2(\bar{u}-\underline{u})} .
$$

The other possibilities,

- $u_{+}-u_{-}>\bar{u}-\underline{u}, u_{-}>\underline{u}$, and $u_{+}>\bar{u}$

- $u_{+}-u_{-}>\bar{u}-\underline{u}, u_{-}<\underline{u}$, and $u_{+}<\bar{u}$,

are treated similarly. $\otimes$

Regarding the general case when both $X_{i}$ and $R_{i}$ have infinite support, the results in the $\mathrm{BF}$ case match those of the LO case:

(i) From the definition of $g_{B F}$ we have $g_{B F}$ decreasing at rate $O\left(u^{-1}\right)$ as $u \rightarrow \infty$. This implies that $g_{L O}(u)-g_{B F}(u) \rightarrow 0$ as $u \rightarrow \infty$; see the right part of Figure 5 .

(ii) $g_{B F}$ is sub-additive; the proof of this fact is similar to the LO one. Hence, $g_{B F}$ is locally linear (increasing) around 0 . Moreover, it can be shown that the linear constants are the same in both the LO and BF cases.

(iii) Items (i) and (ii) imply that the optimal cycle length satisfies $g_{B F}^{\prime}(u)=0$, which can be solved numerically at a cost that grows with the number of roots. 
(iv) Effects of arrival location variability: Here we also have that re-scalings of $X$, such as $c X$, lead to lower detection rate if and only if the scaling constant $c$ is greater than 1 .

The next example illustrates many of the results of this section.

Example 9 Suppose $X_{i} \sim N(0,1)$ and $R_{i} \sim \exp (\rho)$, for $\rho>0$. We have $a_{B F}^{*}=-u / 2$ and completing squares in equations (9) and (8) results in

$$
g_{B F}(u)=\frac{(\Phi(u / 2)-\Phi(-u / 2))-e^{2 \rho^{2}-\rho u}(\Phi(2 \rho+u / 2)-\Phi(2 \rho-u / 2))}{u \rho} .
$$

Figure 5 shows the detection rates for the LO case on top and for the BF case on bottom when $\rho=1$. As expected from Lemma 6 , we observe $g_{B F}(u) \leq g_{L O}(u)$ for all $u \geq 0$. In addition, $g_{B F}(u) \approx g_{L O}(u)$ locally around 0 because there are no reneges there, and $g_{B F}(u) \approx$ $g_{L O}(u)$ for all $u$ sufficiently large because the expected number of detections per cycle converges to $\mu_{R}$ (i.e.: the numerator in Eqs. (3) and (9) converges to $\mu_{R}$ as $u \rightarrow \infty$ ), yet the increasing cycle length causes the detection rate to decrease at speed $O\left(u^{-1}\right)$. The operational take-away is that there is not a significant difference between the LO and BF trajectories when the cycle length is small or large.

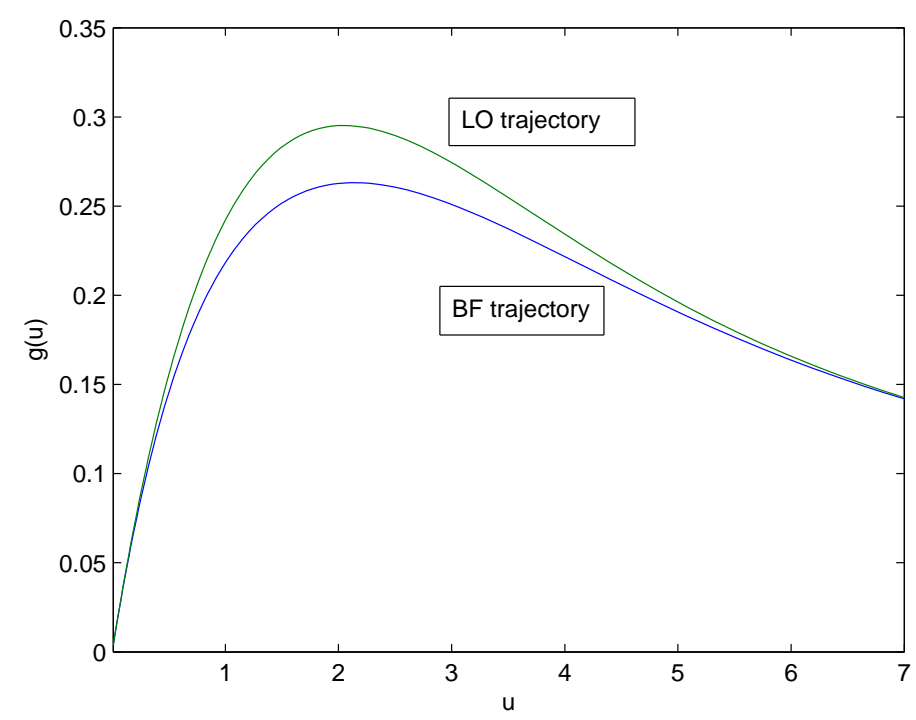

Figure 5: Detection rate for LO and BF trajectories.

Figure 6 presents $u_{B F}^{*}, g_{B F}\left(u_{B F}^{*}\right), u_{L O}^{*}$, and $g_{L O}\left(u_{L O}^{*}\right)$ as a function of the reneging rate $\rho$. We observe in the top part that $u_{B F}^{*} \geq u_{L O}^{*}$ for all $\rho$; the reason for this is that the BF trajectory compensates the reneges at the endpoints with the extra detections provided by a larger trajectory length. (This argument can be made rigorous, but there is not much value in comparing optimal cycle lengths). Regarding the bottom part of Figure 6 we have, as expected from Corollary 1, that $g_{B F}\left(u_{B F}^{*}\right) \leq g_{L O}\left(u_{L O}^{*}\right)$, with the difference $g_{L O}\left(u_{L O}^{*}\right)-g_{B F}\left(u_{B F}^{*}\right)$ being largest for moderate values of $\rho$. In other words, when targets have an expected renege time that is sufficiently small or large, the LO trajectory is not much better than the BF trajectory. 

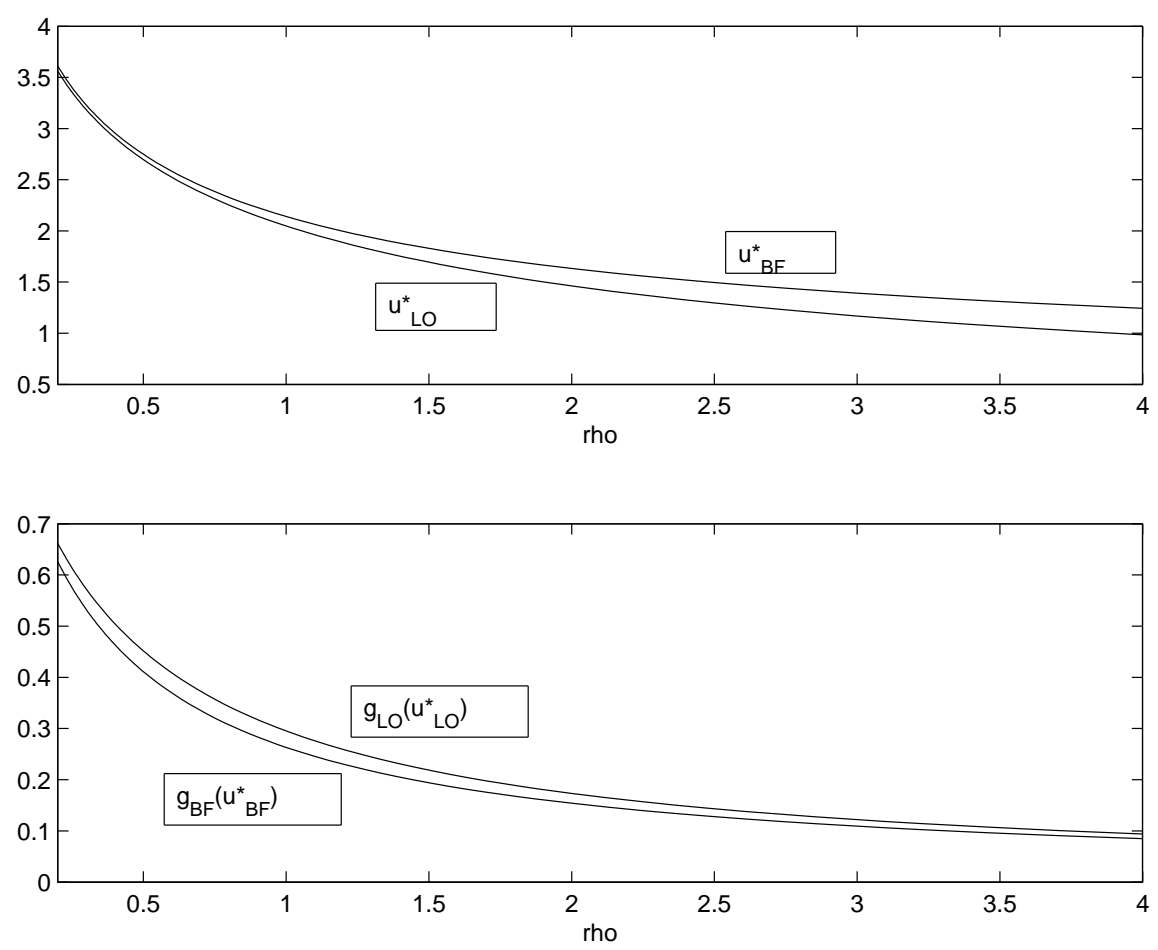

Figure 6: Cycle length and detection rate as a function of reneging rate.

\section{Effects of Velocity and Investigation Time}

Although the baseline scenarios described in the previous sections are idealized, they can be adjusted in ways that better approximate sensor operation in the field. In this section we put forward two such extensions. In the first extension we study the effect of sensor velocity on the long term detection rate. In particular, we consider the tradeoff between visiting a point on the perimeter more frequently, which mitigates the effect of reneging, and missing targets due to decreased detection capability when sensor's velocity increases. In the second extension we study the case where it takes time to investigate each detected target.

Remark 4 The effects of these extensions for both the LO and BF settings are qualitatively similar. In this paper we only present the LO trajectory since it is more tractable.

\subsection{Effects of velocity}

If sensor velocity has no impact on its ability to detect infiltrators that cross its trajectory then it is obvious that the optimal cycle length and detection rate increase with sensor velocity, and maximum velocity is optimal. On the other hand, if high velocity leads to a lower detection capability, low sensor velocity may be optimal. In this section we investigate the effect of this dependency.

Given that the sensor looks at a target, we consider the case where the target is detected with a probability that is non-increasing in the sensor velocity; this case captures the situation where the sensor operator tends to miss targets as the sensor velocity increases. Because 
the detection process in this setting is a thinned version of the detection process $N_{d}(\cdot)$ (see [14], p. 317), in order to maximize detection rate, velocity $v$ and cycle length $u$ should be selected in the LO context so as to maximize

$$
g_{L O}(u, v):=\frac{\eta(v) H(u) \int_{0}^{u / v} \bar{F}_{R}(t) d t}{u / v}
$$

where $\eta(v)$ is the detection probability when the sensor moves at velocity $v$. The thinning of the detection process shows as the $\eta(v)$ term in Equation (12). Naturally, we seek an answer to the following question: What is the (velocity, cycle length) pair that maximizes detection rate? We assume that the detection probability $\eta(v)$ is differentiable for all $v>0$.

Example 10 Suppose $\eta(v)=\eta \leq 1$ is positive and constant for all $v>0$. This is the case where sensor velocity has no impact on the sensor ability to detect targets. We have

$$
g_{L O}(u, v):=\frac{\eta H(u) \int_{0}^{u / v} \bar{F}_{R}(t) d t}{u / v} .
$$

For $u$ arbitrary, $\partial g_{L O}(u, v) / \partial v>0$ shows that going at maximum speed (say $\bar{v}$ ) is optimal. Changing variables, $\psi=u / v$, shows that maximizing $g_{L O}(u, \bar{v})$ is the same as maximizing

$$
\frac{\eta H(\bar{v} \psi) \int_{0}^{\psi} \bar{F}_{R}(t) d t}{\psi} .
$$

Hence, we are in the framework of Section 3, with $\psi$ in place of $u$. Let $\psi^{*}$ be the smallest maximizer in (13), and $u^{*}(\bar{v})=\bar{v} \psi^{*}$. A repeat of the arguments used in Section 3 shows that $u^{*}(\bar{v}) \geq u^{*}$ and $g_{L O}\left(u^{*}(\bar{v}), \bar{v}\right) \geq g_{L O}\left(u^{*}\right)$ if and only if $\bar{v} \geq 1$. In other words, increasing velocity can only improve detection rate.

The analogue of Example 1 is

Example 11 Suppose $0<\bar{r}<\infty$. Then

$$
g_{L O}(u, v)=\left\{\begin{array}{c}
\eta(v) H(u) \frac{\int_{0}^{u / v} \bar{F}_{R}(t) d t}{u / v}, \text { for } 0 \leq u / v \leq \bar{r} \\
\eta(v) H(u) \frac{\mu_{R}}{u / v}, \text { for } u / v>\bar{r}
\end{array}\right.
$$

In the above example, if $H$ is strictly concave then $H(u) / u$ is decreasing for all $u>0$, and so we must have $u^{*} \leq v^{*} \bar{r}$ (cf. Lemma 3 ). The continuation of Example 2 is

Example 12 Suppose that $P(R=\bar{r})=1,0<\bar{r}<\infty$. Then, if $H$ is concave, we have $u^{*}(v)=\bar{r} v$ and

$$
g_{L O}\left(u^{*}(v), v\right)=\eta(v) H(\bar{r}) .
$$

Because $\eta(v) H(\bar{r}) \rightarrow 0$ if $\eta(v) \rightarrow 0$ as $v \rightarrow \infty$, we must have $0<v^{*}<\infty$, so that the optimal velocity satisfies $(\eta(v) H(v \bar{r}))^{\prime}=0$.

The following is an example of practical interest because it treats the case of a uniform distribution of the targets' arrival location along the perimeter. 
Example 13 Suppose $X_{i} \sim U(0, \bar{u})$. For any $v>0$, Example 4 implies that $u^{*}(v)=\bar{u}$, so that

$$
g_{L O}(\bar{u}, v)=\frac{\eta(v) v \int_{0}^{\bar{u} / v} \bar{F}_{R}(t) d t}{\bar{u}} .
$$

Observe that for all $0<v \leq \epsilon, \epsilon>0$, we have

$$
g_{L O}(\bar{u}, v) \leq \epsilon \frac{\eta(0) \mu_{R}}{\bar{u}}
$$

which implies that $g_{L O}(\bar{u}, v) \rightarrow 0$ as $v \rightarrow 0$. Similarly, for all $v>\xi>0$,

$$
g_{L O}(\bar{u}, v) \leq \frac{\eta(\xi) \xi \bar{u} / \xi}{\bar{u}}=\eta(\xi)
$$

shows that $g_{L O}(\bar{u}, v) \rightarrow 0$ as $v \rightarrow \infty$. Therefore, the optimal velocity is $0<v^{*}<\infty$, and can be found by solving $\partial g_{L O}(\bar{u}, v) / \partial v=0$. In this regard, $g_{L O}(\bar{u}, v)$ strictly concave in $v$ would imply the existence of only one such root.

Looking now at the general case, we start by exploring some properties of the optimal (velocity, cycle length) pair.

Lemma 9 Suppose that $\eta(v)$ is non-increasing and goes to 0 as $v \rightarrow \infty$. Then $0<u^{*}<\infty$ and $0<v^{*}<\infty$.

Proof: Observe that for all $u, v>0$ we have

$$
\frac{\int_{0}^{u / v} \bar{F}_{R}(t) d t}{u / v} \leq 1
$$

We study four complementary scenarios: For $0<u<\epsilon, \epsilon>0$, Equation (14) implies $g_{L O}(u, v) \leq H(\epsilon) \eta(0)$. Second, for $0<v<\epsilon, \epsilon>0$, we have $g_{L O}(u, v) \leq \epsilon \eta(0) \mu_{R} / u$. Third, for $u>\xi>0: g_{L O}(u, v) \leq v \eta(0) \mu_{R} / \xi$. Last, for $v>\xi>0$, Equation (14) implies $g_{L O}(u, v) \leq \eta(\xi)$. Sending $\epsilon \rightarrow 0$ and $\xi \rightarrow \infty$ in the above scenarios completes the proof. $\otimes$

The last lemma lets us conclude that the optimal $\left(u^{*}, v^{*}\right)$ pair must be a stationary point

$$
\frac{\partial g_{L O}(u, v)}{\partial u}=0, \text { and } \frac{\partial g_{L O}(u, v)}{\partial v}=0 .
$$

Just like in the LO and BF case, we use numerical optimization to find such stationary point, and select the one for which the detection rate is largest. Finding the optimal $\left(u^{*}, v^{*}\right)$ pair can become numerically costly when there exist many roots that need to be found and compared.

Example 14 Suppose that $X_{i} \sim \exp (1), R_{i} \sim U(0,1)$, and $\eta(v)=\exp (-v)$. Then a direct calculation shows that

$$
g(u, v)=e^{-v}\left(1-e^{-u}\right)(1-.5 u / v),
$$

for $u \leq v$; this constraint is justified by the discussion following Example 11. Solving (15) yields $\left(u^{*}, v^{*}\right)=(.610, .726)$, and $g\left(u^{*}, v^{*}\right)=.128$. We now explain how this .128 fraction is obtained. $P\left(X \leq u^{*}\right)=.457$ is the fraction of targets that arrive to the part of the perimeter that is searched. Of these, $.457 \times \int_{0}^{u^{*} / v^{*}} \bar{F}_{R}(t) d t=.223$ are observed before reneging. Finally, $.223 \times \exp \left(-v^{*}\right) /\left(u^{*} / v^{*}\right)=.128$ is the fraction of targets that is detected. 

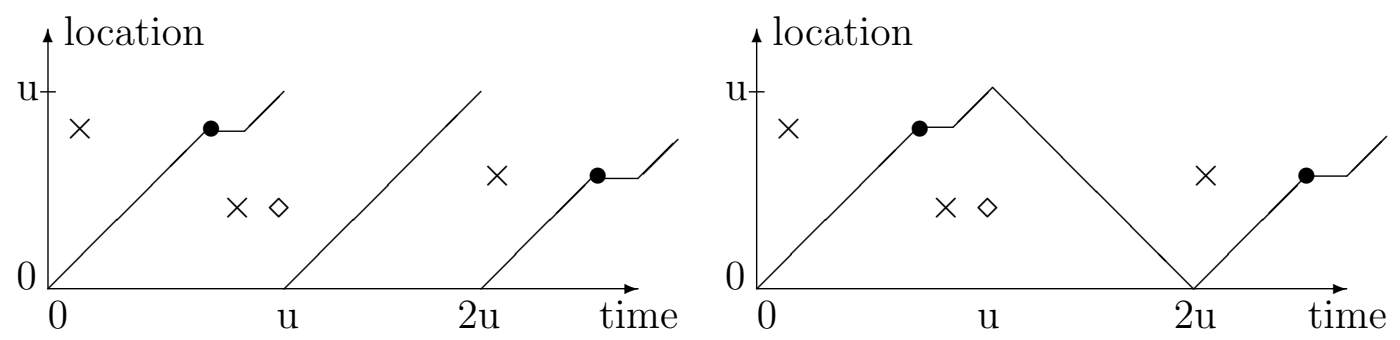

Figure 7: The LO trajectory (left) and BF trajectory (right), subject to investigation time.

\subsection{Investigation time}

So far we have postulated operational scenarios under the assumption that the detection occurs instantaneously. In certain situations, however, targets are investigated by the sensor upon detection, during which time the sensor remains immobile. This type of sensor policy is common when the identification of the target is required before a decision is made as to the necessary reaction, or when the sensor has to escort the target until a response force arrives at the scene. Figure 7 illustrates this scenario when the investigation time is constant.

As in the previous sections, we would like to find a cycle length that maximizes detection rate as $t \rightarrow \infty$. Unfortunately, for a given cycle length $u$, the detection rate cannot be analytically computed, and must be estimated by Monte Carlo simulation. This happens because disjoint intervals do not have independent number of detections, so the detection process is not Poisson anymore. Hence, we must use simulation optimization ideas to find a good cycle length.

To make our developments simple we assume that investigation times are constant and equal to $\theta>0$. The key to approximating detection rate for large values of $t$ is to notice that the number of detections that fall within complete uninterrupted trajectories and the times elapsed between these trajectories are i.i.d. random variables. In other words, the system regenerates whenever a trajectory with no detections occurs.

To get started, let $Z=\left(Z_{i}: i \geq 0\right)$ be a stochastic process with state space on $R^{\infty}$. A realization $Z_{i}=\left(t_{i, 0}, t_{i, 1}, \ldots, t_{i, n}\right)$ says that the $i$ 'th trajectory starts at time $t_{i, 0}$, and the $j$ 'th detection occurs at time $t_{i, j}$, for $j=1, \ldots, n, n$ being the last detection in that trajectory. We set $Z_{0}=(0)$ to indicate that there are no detections in the first (irregular) cycle. Because we know velocity and the endpoints of the patrolled sector, given $Z_{i}$ we also know the location of the arrivals in that cycle. Observe that the $Z_{i}$ 's are, in general, not i.i.d. random variables. However, homogeneous Poisson arrivals imply that $P\left(Z_{n} \in\right.$ $\left.\cdot \mid Z_{n-1}, \ldots, Z_{1}\right)=P\left(Z_{n} \in \cdot \mid Z_{n-1}\right)$, and hence the process $Z$ is Markov. Let us define stopping times $\tau_{1}=\inf \left\{i>0:\left|Z_{i}\right|=1\right\}$, and $\tau_{n}=\inf \left\{i>\tau_{n-1}:\left|Z_{i}\right|=1\right\}$ for $n \geq 2$, where by $\left|Z_{i}\right|$ we denote the cardinality of $Z_{i}$. Observe that the $\tau_{i}$ 's are i.i.d. random variables. Observe that if targets never renege it is possible to have $\tau=\infty$. However, when $0<\mu_{R}<\infty$, we have the following

Lemma 10 If $0<\mu_{R}<\infty$ then $E \tau_{1}<\infty$.

Proof: Assume momentarily that

$$
P\left(\tau_{1}>k\right) \leq\left(1-\exp \left(-\mu_{R}\right)\right)^{k},
$$


for $k \geq 1$. Hence,

$$
E \tau_{1}=\sum_{k=0}^{\infty} P\left(\tau_{1}>k\right) \leq \exp \left(\mu_{R}\right)<\infty .
$$

To complete the proof we use induction to show (16):

$$
P\left(\tau_{1}>1\right)=P\left(\left|Z_{1}\right|>1\right)=1-\exp \left(-H(u) \int_{0}^{u} \bar{F}_{R}(t) d t\right) \leq 1-\exp \left(-\mu_{R}\right),
$$

where the second equality follows from the fact that the process $N_{d}$ is Poisson. A similar argument verifies the inequality

$$
P\left(\left|Z_{k+1}\right|>1|| Z_{k} \mid>1\right) \leq 1-\exp \left(-\mu_{R}\right)
$$

for $k>1$. Assume that $P\left(\tau_{1}>k\right) \leq\left(1-\exp \left(-\mu_{R}\right)\right)^{k}$ for $k>1$. Then,

$$
\begin{aligned}
P\left(\tau_{1}>k+1\right) & =P\left(\left|Z_{k+1}\right|>1,\left|Z_{k}\right|>1, \cdots,\left|Z_{1}\right|>1\right) \\
& =P\left(\left|Z_{k+1}\right|>1|| Z_{k} \mid>1\right) P\left(\left|Z_{k}\right|>1, \cdots,\left|Z_{1}\right|>1\right) \text { (Markov property) } \\
& \leq\left(1-\exp \left(-\mu_{R}\right)\right)\left(1-\exp \left(-\mu_{R}\right)\right)^{k}(\text { by }(17) \text { and the induction hypothesis). }
\end{aligned}
$$

$\otimes$

We will also make use of the following

Corollary 2 If $0<\mu_{R}<\infty$ then $E\left|Z_{k}\right|<\infty$, for $k \geq 1$.

Proof: The proof follows from (17) since $P\left(\left|Z_{k}\right|>1\right)=E\left(P\left(\left|Z_{k}\right|>1|| Z_{k-1} \mid>1\right)\right) \leq$ $1-\exp \left(-\mu_{R}\right)$ implies $E\left|Z_{k}\right|<\infty$. $\otimes$

Lemma 10 and its corollary let us use the Law of Large Numbers for Renewal Processes to conclude that

$$
\frac{\# \text { detections by time } t}{t} \rightarrow g_{I N V}(u),
$$

almost surely as $t \rightarrow \infty$, where

$$
g_{I N V}(u):=\frac{E\left(\sum_{i=1}^{\tau_{1}}\left(\left|Z_{i}\right|-1\right)\right)}{\theta E\left(\sum_{i=1}^{\tau_{1}}\left(\left|Z_{i}\right|-1\right)\right)+u E \tau_{1}} .
$$

The term $\sum_{i=1}^{\tau_{1}}\left(\left|Z_{i}\right|-1\right)$ is the (random) number of detections in a renewal, and $\theta \sum_{i=1}^{\tau_{1}}\left(\left|Z_{i}\right|-\right.$ $1)+u \tau_{1}$ is the (random) renewal time. Lemma 10 and its corollary ensure that $g_{I N V}(u)$ is well-defined. Observe that $g_{I N V}(u) \rightarrow 1 / \theta$ as the target arrival rate goes to $\infty$, for $\mu<\infty$. Although the notation does not reflect it, the right-hand side in (19) depends on cycle length $u$.

Assume that $g_{I N V}$ has a unique maximizer $u_{I N V}^{*}$. Then, because $g_{I N V}(u) \leq g_{L O}(u)$ for all $u>0$, we have $g_{I N V}(u)=O\left(u^{-1}\right)$ and $u_{I N V}^{*}<\infty$. In other words, the search space of the optimal cycle length is bounded. Regarding the optimal cycle origin, when $\bar{r}<u$ it is possible to show that $a^{*}$ is a solution of (1). Unfortunately, the problem is analytically intractable in the general case when $\bar{r}>u$ (possibly $\bar{r}=\infty$ ). We carried out an exhaustive simulation optimization analysis for various distributions of $R$ and $X$ that meet Assumption 
1 , and found that $a^{*}$ is a solution of (1) in all scenarios. This leads us to conjecture that the optimal cycle origin solves (1).

In order to find an estimate of a cycle length that maximizes $g_{I N V}$, we employ the method of Sample Average Approximation (SAA); see chapter 6 of [15] for an overview of SAA, and Theorem 2.1 of [16] for the version of SAA used in this paper. The basic idea of SAA is to maximize $g_{I N V}(u)$ by optimizing (the ratio of) sample averages

$$
\hat{g}_{n}(u):=\frac{\bar{V}_{n}(u)}{\bar{W}_{n}(u)},
$$

where $\bar{V}_{n}(u)=1 / n \sum_{i=1}^{n} V_{i}(u), \bar{W}_{n}(u)=1 / n \sum_{i=1}^{n} W_{i}(u)$, and the $\left(V_{i}(u), W_{i}(u)\right)$ 's are i.i.d. random samples drawn from the same distribution of $\left(\sum_{i=1}^{\tau_{1}}\left(\left|Z_{i}\right|-1\right), \theta\left(\sum_{i=1}^{\tau_{1}}\left(\left|Z_{i}\right|-1\right)\right)+u \tau_{1}\right)$. Although this distribution is generally unknown, we can obtain samples from it by generating a sufficiently large stream of reneging times $R_{i}$, locations $X_{i}$, and arrival times $T_{i}$, and then using discrete event simulation [11].

In order to use SAA, we must have $V(u)$ and $W(u)$ Lipschitz continuous:

$$
\left|V\left(u_{1}, \omega\right)-V\left(u_{2}, \omega\right)\right| \leq \nu(\omega)\left|u_{1}-u_{2}\right|
$$

and

$$
\left|W\left(u_{1}, \omega\right)-W\left(u_{2}, \omega\right)\right| \leq \psi(\omega)\left|u_{1}-u_{2}\right|,
$$

where $\omega$ is a sample path, and $\nu$ and $\psi$ are functions of $\omega$ with finite second moments. It is possible to bound the LHS of (20) by the number of arrivals over the largest regenerative cycle $\tau_{1}$ (amongst the two cycles stemming from selecting $u_{1}$ or $u_{2}$ ), which due to the Poisson arrival assumption and Corollary 2 has a finite second moment. Hence, for $\left|u_{1}-u_{2}\right| \geq 1$, Equation (20) is satisfied. Using the same type of argument, it is possible to bound the LHS of (21), for $\left|u_{1}-u_{2}\right| \geq 1$. Unfortunately, this approach does not work for (20) and (21) when $\left|u_{1}-u_{2}\right|<1$, and our setup is too complex to enable analytical verification of both Lipschitz conditions. To overcome this difficulty, we ran a simulation with a very large number of replications and several choices of parameters $u_{1}$ and $u_{2}$ such that $\left|u_{1}-u_{2}\right|<1$; the simulation results indicate both Lipschitz conditions are satisfied when $\left|u_{1}-u_{2}\right|<1$.

Let $\hat{u}_{n}$ be the cycle length obtained by numerically maximizing $\hat{g}_{n}$. Then, Theorem 2.1 of [16] (a version of the Delta Theorem) ensures that

$$
n^{1 / 2}\left(\frac{g_{n}\left(\hat{u}_{n}\right)-g_{I N V}\left(u_{I N V}^{*}\right)}{\hat{\sigma}_{n}}\right) \Rightarrow N(0,1),
$$

as $n \rightarrow \infty$, where

$$
\hat{\sigma}_{n}^{2}=\sigma_{V}^{2} \frac{1}{\bar{W}_{n}^{2}\left(\hat{u}_{n}\right)}+\sigma_{W}^{2} \frac{\bar{V}_{n}^{2}\left(\hat{u}_{n}\right)}{\bar{W}_{n}^{4}\left(\hat{u}_{n}\right)}-2 \sigma_{V W} \frac{\bar{V}_{n}\left(\hat{u}_{n}\right)}{\bar{W}_{n}^{3}\left(\hat{u}_{n}\right)},
$$

and $\sigma_{V}^{2}=(n-1)^{-1} \sum_{i=1}^{n}\left(V_{i}\left(\hat{u}_{n}\right)-\bar{V}_{n}\left(\hat{u}_{n}\right)\right)^{2}, \sigma_{W}^{2}=(n-1)^{-1} \sum_{i=1}^{n}\left(W_{i}\left(\hat{u}_{n}\right)-\bar{W}_{n}\left(\hat{u}_{n}\right)\right)^{2}$, $\sigma_{V W}^{2}=(n-1)^{-1} \sum_{i=1}^{n}\left(V_{i}\left(\hat{u}_{n}\right)-\bar{V}_{n}\left(\hat{u}_{n}\right)\right)\left(W_{i}\left(\hat{u}_{n}\right)-\bar{W}_{n}\left(\hat{u}_{n}\right)\right)$.

This result provides theoretical support for building asymptotically valid confidence intervals for $g_{I N V}\left(u_{I N V}^{*}\right)$ :

$$
P\left(g_{I N V}\left(u_{I N V}^{*}\right) \in\left[L_{n}, R_{n}\right]\right) \rightarrow 1-\delta,
$$




\begin{tabular}{|l|cccccc|}
\hline \multirow{2}{*}{ Parameter } & \multicolumn{5}{|c|}{ Investigation time $\theta$} \\
\cline { 2 - 7 } & 0.0 & 0.2 & 0.4 & 0.6 & 0.8 & 1.0 \\
\hline$g_{n}\left(\hat{u}_{n}\right) \pm 1.96 \hat{\sigma}_{n} / n^{1 / 2}$ & .295 & $.287 \pm .01$ & $.276 \pm .008$ & $.265 \pm .008$ & $.244 \pm .007$ & $.240 \pm .007$ \\
$\hat{u}_{n}$ & 2.05 & 2.04 & 1.97 & 1.90 & 1.92 & 1.90 \\
$\hat{a}_{n}$ & -1.02 & -1.02 & -.99 & -.95 & -.96 & -.96 \\
\hline
\end{tabular}

Table 1. Detection rate with different investigation times.

as $n \rightarrow \infty$, where

$$
\begin{aligned}
& L_{n}=g_{n}\left(\hat{u}_{n}\right)-z \frac{\hat{\sigma}_{n}}{n^{1 / 2}}, \\
& R_{n}=g_{n}\left(\hat{u}_{n}\right)+z \frac{\hat{\sigma}_{n}}{n^{1 / 2}},
\end{aligned}
$$

and $z$ is selected so that $P(-z \leq N(0,1) \leq z)=1-\delta$.

Example 15 Set $X_{i} \sim N(0,1), R_{i} \sim \exp (1)$, and $n=3000$. For investigation times $\theta=$ $.2, .4, .6, .8$, and 1 , we construct an approximate $95 \%$ confidence interval for $g_{I N V}\left(u_{I N V}^{*}\right)$. The results are summarized in Table 1. As expected, detection rate decreases as investigation time increases. Compared to our result from Example 5, $g_{L O}\left(u_{L O}^{*}\right)=.295$, the simulation results indicate that reasonably small investigation times cause no significant degradation in detection rate. The optimal cycle length $\hat{u}_{n}$ decreases with the investigation time-this happens because investigation time causes more targets to renege before detection, and the perimeter traversed is chopped at both endpoints to counter this fact. The row headed by $\hat{a}_{n}$ shows the optimal origin, as determined by the simulation and optimization results; $\hat{a}_{n}$ does not exactly equal $-\hat{u}_{n} / 2$ due the finite number of regenerative cycles generated during the simulation.

\section{Final Remarks}

In this paper we developed models of border surveillance when infiltrators arrive according to a Poisson process and can renege after a random amount of time. For both leap-to-origin and back-and-forth trajectories, we derived an expression for the detection rate given the reneging, arrival location distribution and the length of the patrolled sector (the decision variable). We extended this analysis to study the effects of sensor velocity and investigation time.

Our analysis suggests that there is not a significant difference in the target detection rate among the two trajectories considered when the cycle length is very small or very large. When each target is investigated upon detection, we find that the cycle length that maximizes detection rate decreases as investigation time increases, in order to compensate for the extra reneging of targets that occurs. 
A basic limitation of our models is that the sensor footprint is one-dimensional. This assumption is a reasonable representation of reality when the size of the sector is significantly greater than the sensor's footprint width.

Given that our modeling assumptions are relatively mild and the operational scenarios we describe are currently being employed (as in, for example, the U.S.-Mexico border; see [1] and [17]), we believe that the sequence of sensor/target configurations we pose in this paper yields valuable insights into the operation of sensor platforms in realistic border control settings.

\section{References}

[1] Christopher Bolkcom. Homeland security: Unmanned aerial vehicles and border surveillance. CRS Report for Congress, 2004.

[2] R. C. Bonner. National border patrol strategy: Message from the commissioner. Washington D.C.: U.S. Customs and Border Protection, Office of Border Patrol and the Office of Policy and Planning, September 2004.

[3] E. Çinlar. An introduction to spatial queues, pages 103-118. CRC Press, 1995. Dshalalow (ed.).

[4] D. J. Daley and D. Vere-Jones. An Introduction to the Theory of Point Processes. Springer, 2nd edition, 2003.

[5] Department of Homeland Security. A review of remote surveillance technology along the US land borders, 2005. Department of Homeland Security Office of Inspector General OIG 06-15.

[6] Fuentes J. R. Finckenauer, J. O. and G. L. Ward. Mexico and the United States: Neighbors confront drug trafficking. http://www.ojp.usdoj.gov/nij/international/trafficking.html, 2001. National Institute of Justice, International Center.

[7] News Hour. U.S.-Mexico border dispute. http://www.pbs.org/newshour/, August 18th 2005.

[8] John D. Kettelle and Daniel H. Wagner. Acoustic effectiveness of specific nuclear submarines. David Taylor Model Basin, 1963.

[9] Bernard O. Koopman. Search and Screening. Office of the Chief of Naval Operations, Operations Evaluation Group Report 56, 1946.

[10] Eric S. Langford. A continuous submarine versus submarine game. Naval Research Logistics Quarterly, 20:405-417, 1973.

[11] A. M. Law and W. D. Kelton. Simulation Modeling and Analysis. McGraw-Hill, New York, 3rd edition, 2000. 
[12] Edward P. Loane and Daniel H. Wagner. Submarine-versus-Submarine Secure Sweep Width Manual. Commander Submarine Group Two and David Taylor Model Basin, Associates Report to Bureau of Ships, 1964.

[13] P. M. Orrenius. Illegal immigration and enforcement along the U.S.-Mexico border: An overview. Economic and Financial Review, (1), 2001.

[14] Sidney Resnick. Adventures in Stochastic Processes. Birkhuser, 1992.

[15] A. Ruszczyński and A. Shapiro. Stochastic Programming, volume 10 of Handbooks in Operations Research and Management Science. Elsevier, 2003.

[16] Alexander Shapiro. Asymptotic analysis of stochastic programms. Annals of Operations Research, 30:169-186, 1991.

[17] The New York Times. U.S. project to secure borders will begin in Arizona desert. http://www.nytimes.com/2006/09/22/us/22border.html, September 22nd 2006.

[18] Alan R. Washburn. On patrolling a channel. Naval Research Logistic Quarterly, 29:609615, 1982.

[19] Alan R. Washburn. Search and Detection. Institute for Operations Research and the Management Sciences, 4th edition, 2002. 